\title{
A pellet launcher tool optimized for the control of edge localized modes in ASDEX Upgrade H-mode plasmas
}

\author{
P.T. Lang* ${ }^{*}$ P. Cierpka, J. Harhausen, M. Kaufmann ${ }^{\dagger}$, J. Neuhauser, \\ C. Wittmann, ASDEX Upgrade Team \\ Max-Planck-Institut für Plasmaphysik, EURATOM Association, \\ Boltzmannstr. 2, 85748 Garching, Germany \\ *Corresponding author; e-mail: peter.lang@ipp.mpg.de; ${ }^{\dagger}$ Presenting author \\ G. Kocsis, J. Sárközi, T. Szepesi \\ KFKI-RMKI, EURATOM Association, P.O. Box 49, H-1525 Budapest-114, Hungary \\ C. Dorner, G. Kauke \\ Fachhochschule Regensburg, Fachbereich Maschinenbau, \\ Galgenbergstr. 30, 93053 Regensburg, Germany
}

\begin{abstract}
A main challenge for fusion research oriented pellet launcher systems is the control of the plasma edge region, especially of the possibly dangerous edge localized modes (ELMs) observed in high performance $\mathrm{H}$-mode plasmas. In order to improve the experimental data base required for extrapolation, a novel cryogenic multi-pellet injection system was developed for ASDEX Upgrade, which is entirely dedicated to control and mitigation of type-I ELMs. Based on the blower gun principle, it is capable of combining high repetition rates up to $143 \mathrm{~Hz}$ and low pellet sizes and velocities. Expected benefits are an enhanced tokamak operational area and the option for more sophisticated experiments investigating the underlying physical mechanisms. This paper reports on design criteria, construction, extensive laboratory tests and first injection into ASDEX Upgrade plasmas.
\end{abstract}




\section{Introduction}

During recent decades, injection of solid, cryogenic hydrogen isotope pellets in tokamaks was mainly aiming at particle fuelling [1]. Pellet fuelling in the high confinement (H-mode) regime has revealed several important physical phenomena showing strong impact on fuelling efficiency and hence plasma performance [2]. The most crucial ones are the curvature induced drift of the high pressure plasmoid forming around the ablating pellet $[3,4]$ and the pellet induced release of edge localised modes (ELMs) [5]. The first one can be utilized to improve the fuelling efficiency by launching pellets from the magnetic high field side (HFS) [6]. The second one was initially regarded as an undesired effect increasing additionally the pellet associated losses in case of low field side (LFS) injection. Hence, all major tokamak experiments converted their pellet launch systems such as to allow for high speed launch from the HFS, often at the expense to sacrificing the LFS launch option.

Meanwhile, ELM triggering by pellets, which turned out to happen for HFS launch as well, has been recognised as potentially useful tool to mitigate type-I ELMs in large fusion experiments. In fact, intrinsic ELMs are becoming a severe threat for first wall components with growing machine size, causing severe melting already on JET divertor targets [7] and they are predicted to cause fast erosion of the ITER divertor [8]. Therefore, it is necessary to eliminate type-I ELMs completely or, at least, to reduced the ELM load to below a critical limit. For the latter, ELM triggering is a viable candidate, since it is generally observed that the total average power loss due to ELMs is essentially constant independent of the ELM frequency $f_{E L M}$, i.e. the energy loss per ELM scales inversely with frequency, $\Delta W_{E L M} \sim \frac{1}{f_{E L M}}$ [9]. Therefore ELM mitigation can be achieved by driving up $f_{E L M}$. But, even if ELM pacing by pellets has become a part of the toolkit for plasma control in ASDEX Upgrade [10, 11], it still needs to be improved in order to minimize undesired side effects like excessive core fuelling and confinement degradation [12]. Indeed, all pacing investigations performed so far employed pellet launchers optimized for fuelling rather than for edge control purposes and there is still ample of headroom for a better adaptation. Moreover, a dedicated ELM control system allows to study the physics behind ELM triggering and the variation of the ELMs dynamics with technical control variables, like pellet size, velocity, frequency and poloidal launch position. Such a dedicated system has been designed and optimised for ASDEX Upgrade as described in the following. 


\section{Design criteria}

The task was to develop a new quasi-continuous pellet injection system adapted for ELM control in ASDEX Upgrade as a supplement to the existing, centrifuge based fuelling system used so far also for pellet ELM triggering studies [13]. Therefore the injection rate, pellet size and velocity were primarily determined by ELM pacing needs, with the constraint to minimize undesired fuelling effect. Consequently, small slow pellets resulting in shallow particle deposition causing little fuelling are prerequisites. A further design requirement was that the system should be sufficiently flexible to enable detailed investigations of the ELM trigger mechanism and scaling, with the given temporal resolution of existing ablation diagnostics, favouring as well a low pellet velocity to achieve high spatial resolution along the pellet path. Furthermore, operation with a high pellet reliability $\varepsilon \equiv\left(\frac{\text { Pellets delivered }}{\text { Pellets requested }}\right)$ was desired, and wanted to stay within the range of available technology as far as possible in order to minimize risks and costs. In effect, it was aimed on the highest possible pellet frequency with the smallest possible particle flux and a pellet velocity as small as possible without loosing control of the pellet track.

\subsection{Pellet size}

From previous experiments [12] it can be concluded that a pellet mass of $m_{P} \approx 10^{18} \mathrm{D}$ atoms might be sufficient for ELM pacing, i.e. about $1 \%$ of typical fuelling pellets, reducing the fuelling to an ignorable amount. However, such a drastic reduction of the pellet size means pellet dimension in the order of $250 \mu \mathrm{m}$, for which pellet production, handling and transfer is beyond our experience. Previous mechanical extrusion units indicated that the ice becomes more fragile with increasing surface/volume ratio. In addition, strong transfer losses due to ice sublimation is to be expected. To guarantee a sufficient pellet delivery efficiency a spare extrusion cryostat system was used delivering pellets with a $2 \mathrm{~mm}$ diameter cross section and set the length to 1 $\mathrm{mm}$, though with this pellet parameters the desired minimal particle flux goal is not yet fully achieved. It is therefore envisage to exchange the current extrusion cryostat system by a new one with a reduced pellet cross section (different diameters optional) in a next step.

\subsection{Pellet velocity}

A pellet velocity in the range of about $200 \mathrm{~m} / \mathrm{s}$ was chosen. This compromises a speed low enough to provide sufficiently low penetration depths and the possibility to follow the radial pellet position with high spatial resolution, but high enough to keep the pellet trajectory in the guiding system under control despite repulsion from ablating gas. In addition, pellet delivery times below some $10 \mathrm{~ms}$ guarantee for sufficiently small response times. Based on these consid- 
erations, the blower gun principle [14] was chosen for pellet acceleration relying on the viscous drag acceleration [15]. If hydrogen is used as driver gas no problem will raise from the gas reaching the torus provided it is weak enough to avoid any extra fuelling. The option to reduce the pellet velocity $v_{P}$ by using a driver gas with a lower sound velocity can be taken provided the parasitic driver gas flux to the torus is kept sufficiently low.

\subsection{Pellet injection rate}

For the pellet repetition rate $f_{P}>100 \mathrm{~Hz}$ was thought to be sufficient for ELM pacing providing a reasonable ratio of driving frequency $f_{P}$ to initial intrinsic ELM frequency $f_{E L M}^{0}$ for a reasonably wide range of ELMy H-mode scenarios in ASDEX Upgrade. In order to meet this requirement, pellet extrusion and acceleration has to happen in parallel. A new scheme, called tic-tac shuttle, was therefore developed, as described below.

\subsection{Injection geometry}

Since the new system will operate in addition to the existing HFS fuelling system and for better diagnostic facilities, the design was laid out for LFS launch. At present, only a straight horizontal mid plane injection line is installed, because of operational simplicity and hence high reliability. At least one additional path is under design and construction with a poloidally downward inclined injection path, hitting the magnetic flux surfaces at a much smaller angle. For the purpose of dedicated physics investigations this means extremely shallow particle deposition and nearly vanishing cross field pellet velocity in scrape-off layer (SOL) and pedestal, when the pellet path becomes nearly tangential. Once different injection lines are installed, different injection tracks can be chosen on a discharge to discharge basis.

\section{System layout}

An overview of the experimental set up is given in fig. 1. The right part shows schematically the new blower gun injector. Pellets are delivered from the injector to the torus entrance port via a $5 \mathrm{~m}$ long Teflon guiding tube with $6 \mathrm{~mm}$ inner diameter. Just outside the entrance valve the pellets can be directed to one of the available injection lines (horizontal only at present). In the following, a more detailed description of the main components will be given.

\subsection{Pellet source}

The pellet source comprises two cryostat systems, one for extrusion and one for storing the ice. Liquid Helium is used for cooling, the total consumption is about $5 \mathrm{l} / \mathrm{h}$. Every cryostat has its own coolant and temperature control system, so extrusion and storage temperature can be chosen and adjusted separately. The D fuel is delivered from a storage volume to the extrusion 
cryostat's freezing cell. Small ice crystallites are produced at a pressure of about 2 bar and a temperature of 5 to $10 \mathrm{~K}$. Before extrusion is started compression and annealing by a tempering pulse lasting for about $20 \mathrm{~s}$ takes place, this was found to improve ice quality and pellet stability. Finally, at a sample temperature of $15 \mathrm{~K}$ ice extrusion at an averaged extrusion speed of about 1 $\mathrm{mm} / \mathrm{s}$ is performed through the circular $\emptyset 2 \mathrm{~mm}$ nozzle by applying a pressure of $14 \mathrm{bar}$ on the piston $(\varnothing 6 \mathrm{~mm})$. The ice rod is extruded into the circular 90 degree bent storage cryostat, the length of the single storage channel and hence of the stored ice rod is $124 \mathrm{~mm}$. A light barrier mounted at the bottom of the storage cryostat indicate filling is finished and stops extrusion. The ice reservoir can be kept there if required for up to one hour at a temperature of about 5 $\mathrm{K}$. If a pellet request arrives, the ice rod is shifted out at the bottom of the storage cryostat by a mechanical lever. The single step width is $0.5 \mathrm{~mm}$, every step requires $1 \mathrm{~ms}$; after the shift a pause of $2 \mathrm{~ms}$ is required to bring the vibrating ice rod to rest. Within this boundary conditions, any sequence is accepted, it is e.g. possible to interrupt a feeding/pellet sequence and/or change the feeding/pellet rate.

\subsection{Acceleration unit}

The core part of the injection system is the shuttle. Its task is to allow for pellet feed in and acceleration in parallel along two lines in turn. The shuttle consists of an aluminium frame with an inlay of Vespel and two permanent magnets at both ends. It is mounted moveable on a slide and driven to the end positions by two coils, one pushing and the other one pulling and capturing the shuttle in turn. Fixation at the alternating positions is supported by two Neodymium permanent magnets. Two small bores of $\emptyset 2 \mathrm{~mm}$ are provided in the shuttle to accept the pellet, bring it to the starting position for the acceleration and acting as part of the barrel. With the shuttle in an end position, one of the bores is located in front of the storage cryostats exit and can be filled from the ice rod. Pellet cutting from the rod is achieved when the shuttle is moving over to the alternate position. The bore just filled now arrives in its firing position while the other one is shifted from its firing position to the common filling position. As can be seen from the schematic drawing of the unit in figure 1, the common filling position opposite to the extrusion cryostat (drawn on the same side in figure 1 for simplicity) bottom is located between the firing positions opposite to the two gas valves. In its firing position, the filled caliber completes the acceleration barrel now consisting of the driver pipe at the top of the gas valve nozzle, the caliber and the onset of the guiding tube. The accelerating gas pulse is initiated by a short opening (1.2 - $1.5 \mathrm{~ms}$, pressure dependent) of the fast valve. The gas streams from the nozzle into 
the evacuated multi segmented barrel causing viscous drag acceleration [15] of the pellet to a velocity typically significantly below the gas sound speed. Pre-selecting the appropriate driver gases species and pressure yields acceleration to the requested $v_{P}$ value. The two initial parts of the guiding tube open out finally into the single main tube after $33 \mathrm{~cm}$. To avoid excessive pellet evaporation (especially when operation at low rates or when interrupting a sequence and the last pellet remains in the shuttle) the shuttle is cooled. However, too strong cooling can impact adversarial on the shuttle's gentle, smooth and precise motion. Best over all performance was achieved at a shuttle temperature of about $85 \mathrm{~K}$. Even with the shuttle thus operating at a temperature far above the sublimation temperature of $\mathrm{D}$ (about $18 \mathrm{~K}$ in the pressure range), the Leidenfrost effect [16] avoids too fast pellet evaporation.

\subsection{Pumping and transfer system}

Although the Leidenfrost effect reduces the release of gas by pellet sublimation, ablating pellet material and the driving gas can form a severe threat for stable operation. The gas pressure in the main vessel has to be kept below 0.1 Pa to prevent the onset of significant convective heat flux to the cryostat systems. Once the heat load carried by the exhaust gas approaches a critical limit strong evaporation from the stored ice rod sets in leading into a vicious circle of further enhancing the exhaust gas pressure increasing in turn the heat flux even more. Finally temperature destabilisation and a rapid loss of the ice inventory takes place. Keeping the main vessel pressure sufficiently low even in case of very low pellet repetition rates and/or for frequently interrupted pellet sequences a combined approach relying on strong pumping and sealing off the gas/tube system was made. For the sealing of the gas system at the multi segmented acceleration barrel some restrictions apply. Tightening at the shuttle interfaces too strong imposed unacceptable high damping of its motion. So, a certain amount of leaking gas escaping into the main vessel body was inevitable and has to be removed by sufficient pumping. This was achieved by two turbo molecular pumps each of $360 \mathrm{l} / \mathrm{s}$ pumping capability mounted at separate main vessel ports. Additional pumps of the same type are employed for evacuation of the expansion tank and the selector (one at each). The main fraction of driving gas can be separated and exhausted already via the expansion tank before sending the pellet into the main part of the ex vessel guiding system. Remains of the driver gas flowing into the guiding tube and gas released from the pellet body along its passage are scraped off in the selector unit acting as well as a second expansion tank. The pellet guiding system is composed from small Teflon tubes with $\emptyset 6 \mathrm{~mm}$. The main ex vessel section spanning from the expansion tank to the selector 
is $5 \mathrm{~m}$ long and forms a rather high resistivity for any parasitic gas flow. The torus entrance valve forms a mechanical, vacuum and electrical barrier between the torus vessel and the pellet system. It is kept closed unless gated open during an injection sequence provided the torus protection system [17] checking all relevant system parameters validated this request.

\subsection{Diagnostics}

The pellet system is equipped with numerous permanent intrinsic diagnostics, several additional ones have been used during the operation in the test bed. Temperature sensors embedded in the cryostat systems and the shuttle allow for control and supervising pellet production and storage. A conventional video system is employed for the observation of ice extrusion providing a quality control of the ice rod.

For characterisation of the system the main pellet parameter $m_{P}, v_{P}$ and $\varepsilon$ are measured. Two light barriers performing a time-of-flight measurement determined the initial $v_{P}$ just after the acceleration. Additional light barrier(s) at end of the main ex-vessel transfer tube (single one at the torus, two for a full local $v_{P}$ measurement in the test bed) acted as pellet flyby monitors. Measuring $m_{P}$ in the test bed was done both at the beginning and the end of the main transfer tube by laser flash shadowgraphs. An additional value for the transferred pellet mass was derived by measuring the released gas amount when firing and destroying pellets in a test bed target tank with calibrated volume. Besides indications from the different light barriers, successful pellet arrival was detected with still higher accuracy by an acoustic impact shock sensor in the test bed target tank. After the transfer of the pellet injection system to the torus hall operation of several intrinsic diagnostics was not possible anymore. However, for injection experiments the well diagnosed ASDEX Upgrade plasma [18] itself provided a powerful diagnostics. The $D_{\alpha}$ emitted from the pellet and its surrounding region acted as pellet monitor and delivered an (uncalibrated) mass ablation rate [19]. Cameras measure the pellet trajectory and penetration depths. The impact of the pellet injection on the electron density profile was measured by a DCN laser interferometer and a Thomson scattering system.

\section{Results}

\subsection{Test bed experiment}

The intention of the test bed operation was to characterize and optimize the operational parameter range with respect to the main pellet parameters. The initial $m_{P}=1.6 \times 10^{20} \mathrm{D}$ is already set by the hardware used. This mass is known more than sufficient for ELM triggering in a typical ASDEX Upgrade type-I ELMy H-mode, so it had just to be ensured mass losses occur- 
ring during pellet acceleration and transport are not causing pellet erosion so strong that pellet disintegration sets in. Acceptable operation requires a $\varepsilon$ value fairly close to unity, hence the maximum achievable $f_{P}$ is set by the construction boundary conditions and the requirement to stay still at sufficiently high $\varepsilon$ values. In the end, $v_{P}$ turned out the only remaining free parameter to choose/adjust. In general, this can be done for the applied approach by altering the driver gas pressure, driver gas species, injection geometry parameters like barrel length or the diameter ratio pellet/barrel [15]. Of course the latter two are already fixed by the set up, only the first two can be used for $v_{P}$ adaptation. As driving gases He was used for safety reasons performing pressure scans of the driver gas in the reservoir from 1 to 9 bar. Results are shown in figure 2 where achieved $v_{P}$ values are plotted versus reservoir pressure. In all cases, pellets were fired in sequences of $f_{P}=40 \mathrm{~Hz}$. Clearly as prescribed by the viscous drag acceleration model pellet velocities in the sub-sonic range are observed with increasing $v_{P}$ for raising reservoir pressure. However, stagnation seems to set in already about $v_{P}=300 \mathrm{~m} / \mathrm{s}$ at a level below the expected one. This is clearly an advantage for the envisaged operation. The reason might be friction of the pellet or the transient nature of the propellant gas puff. Further investigations using different gas species and according modelling in order to clarify this question are under way.

When approaching the highest reasonable possible pellet repetitions rates, the resulting pellet velocities for a given gas configuration starts to shrink. Shown in figure 3 are as an example achieved $v_{P}$ values for a given gas parameter set (He propellant gas at 3 bar) plotted versus the pre-selected $f_{P}$ value. Operation with increasing $f_{P}$ requires moving of the shuttle already before the gas pulse has ended scraping off more and more of gas pulse tail. With the gas pulses becoming shorter and shorter, significant parts of the driver gas have already passed the pellet before it reached the end of barrel. Hence, this velocity degradation is due to the fact the effective acceleration time and hence the effective barrel length starts to shrink. The highest value $f_{P}$ at which the system can be reasonably operated was chosen $\frac{1}{7 \times 10^{-3} s} \approx 143 \mathrm{~Hz}$ despite the mild but still acceptable reduction in velocity.

Besides the pellet velocity, the reliability is affected as well by increasing $f_{P}$. There is some very mild degradation observed for the delivery efficiency above $f_{P}=110 \mathrm{~Hz}$ from about 0.9 to about 0.8 . It seems shortening the pellet firing sequence cycle imposes some loss of the driving forces required for the acceleration and might result in pellet loss. In addition, pellet damage or even destruction might take place in some causes during the feed in process since the pellet is not yet in position or at rest when cutting or acceleration is initiated. 


\subsection{First application at ASDEX Upgrade}

At the successful end of the test bed campaign, the range of operational system parameters was fixed and the system thereupon validated for operation at ASDEX Upgrade. Transferred to ASDEX Upgrade and integrated in its safety and control system, here the very first tests of pellet injection and ELM pacing with the new launcher system are reported. Pellet injection was performed on a piggy-back basis during H-mode phases developing low ELM frequencies. Faultless operation of the entire system is demonstrated by the successful arrival of sound pellets in the plasma. An example is shown in figure 4, displaying a half frame (exposure time $20 \mathrm{~ms}$ ) extracted from the torus wide angle video recording system. Two ablation clouds from pellets launched during a $f_{P}=100 \mathrm{~Hz}$ train can be seen close to the designated intersection point of pellet trajectory and plasma separatrix. From the cigar shaped form of both ablation clouds blow up displayed as insert - intact pellet arrival can be concluded.

Although test have proven that the new system is essentially working, it was found reliability to deliver intact pellets is significantly smaller than in the test bed.

Despite pellet fragmentation and destruction hampering the first short experimental approach, already interesting physics investigations on ELM triggering could be performed. One example is shown in figure 5, displaying the impact of three pellets injected during a type-I ELMy Hmode phase. Obviously, as indicated by the pellet ablation $D_{\alpha}$ monitor and the measured line averaged density along a line of sight through the plasma edge region, pellets had different size. The first and presumably largest pellet (note saturation of pellet monitor) clearly triggers a strong ELM and also causes significant perturbation of the background plasma (indicated by the strong pressure pulse to the outer divertor displayed by the $D_{\alpha}$ ELM monitor and the MHD perturbation, both lasting about $2 \mathrm{~ms}$ ). The last pellet triggers an ELM still clearly visible on both monitor signals, but with an almost negligible perturbation of the plasma. With this respect, the mass of this pellet can be considered as ideal for pacing purposes. The pellet in between has still some impact on the MHD behaviour but fails to release a full size ELM. This can be a first hint pellets too small and/or penetrating not sufficiently deep cannot serve as ELM pacer. It is understood first results shown here are not yet sufficient for drawing reliable conclusion but very nicely demonstrate already the potential of the new injector.

Approaches to enhance on this during an in-situ commissioning came to an end by the abrupt premature stop of the AUG C2006 campaign. Analysing the reduced reliability in operation 
with respect to test bed investigations showed a small leakage causing some impurities in the ice reduced pellet stability. As well, a reduction in pellet size was attempted for operation by reducing the initial an tested pellet length to one half. This caused most probably insufficient pellet stability for a cylindrical body with a diameter/length ratio of 4 . With both potential faults removed and an enhanced video stand at the torus entrance port installed in-situ tests were performed indicating the system operating again with full performance. System commissioning is commenced with the aim to have it at disposal with full performance right at the start of the C2007 campaign.

\section{Conclusions}

A new pellet injection system has been developed for ASDEX Upgrade entirely dedicated to edge control purposes. With its small pellet size a reduction of the undesired fuelling is achieved, further upgrading of the system is under way. A new tic-tac feeding and acceleration scheme gives access to very high repetition rates beyond $100 \mathrm{~Hz}$ as required for ITER relevant pacing experiments. With slow pellet velocities in the range $100-300 \mathrm{~m} / \mathrm{s}$ and LFS launch it also allows for dedicated investigations on the ELM dynamics. Further extensions enabling different launch positions are under construction. First successful launch of intact pellets into ASDEX Upgrade was already demonstrated, however in-situ optimization of the performance got stuck due to the early end of the C2006 campaign. An ongoing off-line approach keeping the system at the experiment aims to resume operation at full potential with the restart of the C2007 campaign. Together with the fuelling system still operational, pellet and hence ELM pacing beyond $200 \mathrm{~Hz}$ as well as a direct comparison of different launch sites becomes possible by then.

\section{References}

[1] MILORA, S. L. et al., Nucl. Fusion 35 (1995) 657.

[2] BAYLOR, L. et al., Nucl. Fusion 32 (1992) 2177.

[3] KAUFMANN, M. et al., Nucl. Fusion 26 (1986) 171.

[4] MÜLlER, H. W. et al., Phys. Rev. Lett. 83 (1999) 2199.

[5] LANG, P. T. et al., Nucl. Fusion 36 (1996) 1531, Corrigendum in Nucl. Fus. 36 (1996) 153. 
[6] LANG, P. T. et al., Phys. Rev. Lett. 79 (1997) 1487.

[7] PAMELA, J. et al., Nucl. Fusion 45 (2005) S63.

[8] FEDERICI, F. et al., Plasma Phys. Controlled Fusion 45 (2003) 1523.

[9] HERRMANN, A., Plasma Phys. Controlled Fusion 44 (2002) 883.

[10] HORTON, L. D. et al., 20 $0^{\text {th }}$ IAEA Conf., Vilamoura 2004, EX/P3-4 .

[11] KALLENBACH, A. et al., J. Nucl. Mater. 337-339 (2005) 732.

[12] LANG, P. T. et al., Nucl. Fusion 43 (2003) 1110.

[13] LANG, P. T. et al., Rev. Sci. Instrum. 74 (2003) 3974.

[14] COMBS, S., Rev. Sci. Instrum. 64 (1993) 1679.

[15] GILLIARD, R. et al., Rev. Sci. Instrum. 52 (1981) 183.

[16] LEIDENFROST, J. G., De aquae communis nonnullis qualitatibus tractatus, Duisburg, 1756.

[17] MERTENS, V. et al., Fusion Science and Technology 44 (2003) 593.

[18] HERRMANN, A. and GRUBER, O., Fusion Science and Technology 44 (2003) 569.

[19] MC NEILL, D., J. Nucl. Mater. 162-164 (1989) 476. 


\section{Figure captions}

Figure 1: Overview of the experimental set up. Left (part in box to scale): Poloidal crosssection of the tokamak ASDEX Upgrade with a typical plasma configuration foreseen for ELM investigation experiments; pellet injection paths: horizontal (existing) and tilted (planned) LFS launch with the new system, fuelling trajectory (operational). Right (schematic only): Pellet source consisting of extrusion and storage cryostat. Pellet feed in to shuttle by a stepper motor driven lever. Blower gun injector delivering pellets to the torus, at the torus entrance port the connection to the selected injection line is established.

Figure 2: Pellet velocity versus propellant gas pressure for Helium driver gas. All pellets fired in sequences at a repetition rate of $40 \mathrm{~Hz}$.

Figure 3: Pellet velocity versus pellet repetition rate (Helium driver gas at 3 bar). Beyond 110 $\mathrm{Hz}$ a mild reduction in velocity sets in due to shortening of the acceleration gas pulse.

Figure 4: Video recording (half frame, exposure time $20 \mathrm{~ms}$ ) of two pellets launched at $100 \mathrm{~Hz}$ repetition rate into an ongoing ASDEX Upgrade plasma discharge.

Figure 5: First preliminary results from ELM trigger investigations. Sequence of three pellets of presumably different size (note saturation of pellet monitor for first pellet). Different pellet mass can result in a changed ELM characteristics. First (largest) pellet triggers a clear ELM and imposes significant plasma perturbation, third (medium size) just triggers an ELM, second (smallest) fails to produce full size ELM. 